\title{
Experimental Observation of Optical Frequency Combs in Doubly Resonant Second Harmonic Generation
}

\author{
Iolanda Ricciardi ${ }^{1}$, Simona Mosca $^{1}$, Maria Parisi ${ }^{1}$, Pasquale Maddaloni ${ }^{1}$, Paolo De Natale ${ }^{2}$, \\ Miro Erkintalo ${ }^{3}$, François Leo ${ }^{4}$, Tobias Hansson ${ }^{5}$, Ady Arie $^{6}$, Stefan Wabnitz $^{1,7}$, and Maurizio De Rosa ${ }^{1}$ \\ 1. CNR-INO, Istituto Nazionale di Ottica, Via Campi Flegrei 34, I-80078 Pozzuoli (NA), Italy \\ 2. CNR-INO, Istituto Nazionale di Ottica, Largo E. Fermi 6, I-50125 Firenze, Italy \\ 3. The Dodd-Walls Centre for Photonic and Quantum Technologies, Department of Physics, The University of Auckland, \\ Auckland 1142, New Zealand \\ 4. OPERA-Photonics, Université libre de Bruxelles, 50 Avenue F. D. Roosevelt, CP 194/5, B-1050 Bruxelles, Belgium \\ 5. Department of Physics, Chemistry and Biology, Linköping University, SE-581 83 Linköping, Sweden \\ 6. School of Electrical Engineering, Iby and Aladar Fleischman Faculty of Engineering, Tel-Aviv University, Tel-Aviv, Israel \\ 7. Dipartimento di Ingegneria dell'Informazione, Elettronica e Telecomunicazioni, Sapienza Università di Roma-Via Eudossiana 18, \\ I-00184 Roma, Italy
}

Continuously-driven microresonators, whose nonlinear response is dominated by the third-order Kerr nonlinearity, have proven to be valid alternatives to comb sources based on femtosecond mode-locked lasers [1]. More recenlty, the direct generation of optical frequency combs (OFCs) entirely through quadratic interactions has also been demonstrated in singly resonant cavity second harmonic generation (SHG) and in cw pumped nearly degenerate optical parametric oscillation [2,3]. Interestingly, theoretical studies on doubly resonant cavity SHG predicted the emergence of OFCs with a much lower threshold with respect to the singly resonant configurations, as well as rich nonlinear dynamics [4].

Here we report on the experimental realization of OFC generation in a doubly resonant cavity SHG system. Our experiment is based on a lithium niobate nonlinear crystal placed in a traveling-wave optical cavity, pumped by a cw Nd:YAG laser emitting $0.5 \mathrm{~W}$ at $1064 \mathrm{~nm}$ (Fig. 1(a)). The cavity is resonant for frequencies around both the fundamental pump and its second harmonic at $532 \mathrm{~nm}$, while the nonlinear crystal is a $15-\mathrm{mm}$-long, $5 \%$ $\mathrm{MgO}$ - doped periodically poled lithium niobate sample, quasi phase-matched for SHG. An intracavity adjustable silica window allows to separately set the detunings $\Delta_{1}$ and $\Delta_{2}$ of the pump and its second harmonic from the corresponding nearest cavity resonances, respectively. The cavity is locked to the pump laser frequency by the use of a piezoelectric actuator, according to the Pound-Dever-Hall offset locking technique, thanks to an orthogonally polarized auxiliary beam that is phase modulated and coupled to the cavity in the opposite direction of the pump beam. This scheme permits to achieve stable cavity locking with detunings up to several cavity linewidths, and to observe a large variety of comb regimes, with different teeth spacing and spectral span, as shown in Fig. 1. The lowest experimental threshold for comb formation is around $10 \mathrm{~mW}$, showing the possibility to significantly reduce the input pump power with respect to singly resonant configurations with threshold around $100 \mathrm{~mW}$ [2], and also to diminish photothermal effects. In this regard, an extended theoretical model, which includes thermo-optical nonlinearities, has been developed and will be presented.

(a)

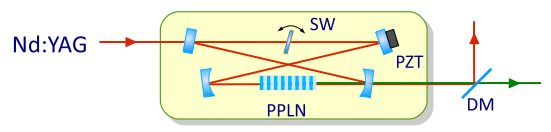

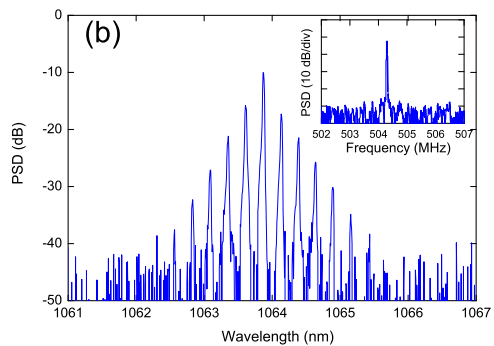

Fig. 1 (a) Scheme of the four-mirror traveling-wave cavity for SHG. PPLN, periodically poled lithium niobate crystal; SW, silica window; DM, dichroic mirror; PZT, piezoelectric actuator. (b) Optical spectrum of the comb emission around the pump frequency, with input power $P_{\text {in }}=300 \mathrm{~mW}, \Delta_{1}=-4$, while the second harmonic field is far from resonance. The inset shows the RF spectrum around the pump frequency, with a beat note at the cavity free spectral range indicating a secondary comb structure, not resolved by the optical spectrum analyzer, around the primary comb teeth. (c) Optical spectrum of the comb emission around the pump frequency, with $P_{\text {in }}=300 \mathrm{~mW}, \Delta_{1}=-2, \Delta_{2}=2 \Delta_{1}$. Note: $\Delta_{1}$ and $\Delta_{2}$ are normalized to the half width at half maximum of the cavity resonance.

\section{References}

[1] P. Del'Haye, A. Schliesser, O. Arcizet, T.Wilken, R. Holzwarth, and T. J. Kippenberg, "Optical frequency comb generation from a monolithic microresonator," Nature 450, 1214 (2007).

[2] I. Ricciardi, S. Mosca, M. Parisi, P. Maddaloni, L. Santamaria, P. De Natale, and M. De Rosa, "Frequency comb generation in quadratic nonlinear media," Phys. Rev. A 91, 063839 (2015).

[3] S. Mosca, M. Parisi, I. Ricciardi, F. Leo, T. Hansson, M. Erkintalo, P. Maddaloni, P. De Natale, S. Wabnitz, and M. De Rosa, "Modulation instability induced frequency comb generation in a continuously pumped optical parametric oscillator," Phys. Rev. Lett. 121, 093903 (2018).

[4] F. Leo, T. Hansson, I. Ricciardi, M. De Rosa, S. Coen, S. Wabnitz, and M. Erkintalo, "Frequency-comb formation in doubly resonant second-harmonic generation," Phys. Rev. A 93, 043831 (2016). 